\title{
A MLS-based lattice spring model for simulating elasticity of materials
}

\author{
Gao-Feng Zhao ${ }^{1}$, Jiannong Fang ${ }^{2 *}$, Jian Zhao ${ }^{1}$
}

1 Ecole Polytechnique Federale de Lausanne (EPFL), Laboratory of Rock Mechanics, Station 18, CH-1015 Lausanne, Switzerland

2 Ecole Polytechnique Federale de Lausanne (EPFL), Laboratory of Engineering and Environmental Geology, Station 18, CH-1015 Lausanne, Switzerland

\begin{abstract}
A MLS-based lattice spring model is presented for numerical modeling of elasticity of materials. In the model, shear springs between particles are introduced in addition to normal springs. However, the unknowns contain only particle displacements but no particle rotations. The novelty of the model lies in that the deformations of shear springs are computed by using the local strain obtained by the moving least squares (MLS) approximation rather than using the particle displacements directly. By doing so, the proposed lattice spring model can represent the diversity of Poisson's ratio without violating the requirement of rotational invariance. Relationships between micro spring parameters and macro material constants are derived from the Cauchy-born rules and the hyperelastic theory. Numerical examples show that the proposed model is able to reproduce elastic solutions obtained by finite element methods for problems without fractures. Therefore, it is capable of simulating solid materials which are initially continuous, but eventually fracture when critical stress and/or displacement levels are reached. A demonstrating example is presented.
\end{abstract}

Keywords: lattice spring model; moving least squares; rotational invariance; elasticity 
* Corresponding author: Email: jiannong.fang@epfl.ch

Tel: +41 2169 32357; Fax: +41216936330

\section{Introduction}

To model a fracture processes in solid, a family of methods coined as lattice models (LMs) have been developed. They are based, in principle, on the atomic lattice models originated from condensed matter physics. In these models, material is represented by a system of discrete units (e.g. particles) interacting via connecting elements. These discrete units are much coarser than the true atomic ones and may represent larger volumes of heterogeneities such as grains or clusters of grains. Compared to a true lattice model, the use of coarse lattices in lattice models dramatically reduces the number of degrees of freedom, and hence makes simulation of continuum systems affordable for medium-sized computers. Lattice models are more suitable for modeling fracture of materials than conventional finite element methods (FEMs) because the former ones simulate fracture by either simply removing connecting elements that exceed the strength or successively degrading their mechanical properties according to cohesive laws. The spatial cooperative effects of crack formation and heterogeneities can be easily investigated through the use of LMs [1, 2].

There exist two different types of lattice models. In the first type models, the material is discretized as a network of springs or beams whose geometry is not related to the actual internal geometry of the material. Here the discrete units are merely lattice sites (nodes). This type of models can be further classified into lattice spring [3-22] and lattice beam [23-26] models according to the number of degrees of freedom per node and the mechanical properties of connecting elements. In a lattice 
spring model (LSM), the unknowns are the nodal displacements and the connecting elements are one-dimensional springs. In a lattice beam model, the unknowns are the nodal displacements and rotations and the connecting elements are beams transferring normal forces, shear forces and bending moments. The second type models are based on the discrete element method originally developed for granular media with contact modeling [27]. For example, the rigid body-spring network model developed by Kawai [28] subdivides the material into rigid particles interconnected along their boundaries through normal and shear springs. It introduces additional rotational degrees of freedom on each particle and hence can be viewed as discretization of a micropolar continuum. Models in this category also include that of Zubelewicz and Bažant [29], the confinement-shear lattice model of Cusatis et al. [30], the bonded-particle model [31], the simple deformable polygonal discrete element model [32], and etc. Recently, new models of the mixed type have emerged, e.g. the explicit quasi-static lattice model [33] and the hybrid lattice particle modeling approach [34].

This article focuses its attention on LSM. The springs usually form a regular network such as triangular lattice in 2D and cubic lattice in 3D. The origin of LSM may trace back to Hrennikoff [3]. The simplest LSM is the normal force model in which only central force interactions (normal springs) are considered. The normal force model has been extensively applied to investigate the elastic and failure properties of a disordered medium [4-9] or the fractal properties of cracks [10-12]. It is also frequently used to the study of fracture or other issues of material sciences [13-15]. However, for the normal force model, it is known that Poisson's ratio obtained by the model approaches, in the limit of an infinite number of particles, a fixed value, namely, 0.25 for 
three-dimensional cases and 0.33 for two-dimensional cases. Such restriction is not suitable for many materials and it can be overcome by introducing non-central force interactions (shear springs) between particles. Hassold and Srolovitz [16] proposed a method to modify the Poisson's ratio by introducing a harmonic potential for rotation of bonds from their initial orientation. Here bonds denote the connecting elements between particles. A non-central two-body interaction limiting the rotational freedom of bonds is introduced in the Born spring model $[17,18]$ to allow a broad choice of Poisson's ratio. Nevertheless, rotational invariance of the models can only be recovered if a three-body interaction is considered. The Kirkwood-Keating spring model [19-22] introduces angular springs to penalize the angular variations between the contiguous bonds incident onto the same node. It is worth mentioning that LSMs are a close relative of FEMs. It has been shown that LSMs are algebraically equivalent to simple FEMs [19, 35]. A finite element mapping procedure has also been proposed to define the spring network representations of solids with an attempt to reproduce all possible material's parameters [36]. Literature review on the development of LSMs can be found in $[37,38]$.

The present paper proposes an alternative MLS-based lattice spring model, which allows one to overcome the restriction on Poisson's ratio while preserving the rotational invariance. The model presented in this paper is two-dimensional and it includes a normal spring and a shear spring for each pair of particles. The deformation of the springs is evaluated by using the local strain rather than the particle displacements. It will be shown that this technique makes the model rotationally invariant. The local strain is calculated via the moving least squares method (MLS) or by using the constant strain triangular element. Firstly, the proposed model and associated numerical techniques 
are described. Secondly, the relationship between spring stiffnesses and elastic constants are derived. Then, the model is validated by numerical simulations of two elastic problems, the effect of negative shear stiffness is studied, and the importance of rotational invariance is revealed. Finally, an example showing the application of the model for fracture simulation is presented.

\section{The model}

The proposed lattice spring model is illustrated in Figure 1 in which the material is represented by a distribution of particles linked through bonds. Each bond includes one normal spring and one shear spring. The bond stiffness matrix is of the form

$$
\mathbf{K}_{\text {bond }}=\left[\begin{array}{cc}
k_{\mathrm{n}} & 0 \\
0 & k_{\mathrm{s}}
\end{array}\right]
$$

where $k_{\mathrm{n}}$ is the normal stiffness and $k_{\mathrm{s}}$ is the shear stiffness. Assume the strain of the bond is $[\boldsymbol{\varepsilon}]=\left[\begin{array}{ll}\varepsilon_{x x} & \varepsilon_{x y} \\ \varepsilon_{x y} & \varepsilon_{y y}\end{array}\right]$, then the normal and shear deformation of the bond can be expressed as

$$
\begin{gathered}
\hat{u}^{n}=([\boldsymbol{\varepsilon}] \cdot \mathbf{n} l) \cdot \mathbf{n} \\
\hat{u}^{s}=([\boldsymbol{\varepsilon}] \cdot \mathbf{n} l-(([\boldsymbol{\varepsilon}] \cdot \mathbf{n} l) \cdot \mathbf{n}) \mathbf{n}) \cdot \mathbf{n}^{\prime}
\end{gathered}
$$

where $l$ is the original length of the bond, $\mathbf{n}$ is the normal vector of the bond which is $\mathbf{n}=\left(n_{x}, n_{y}\right)^{T}$ and $\mathbf{n}^{\prime}=\left(-n_{y}, n_{x}\right)^{T}$ is the unit vector perpendicular to $\mathbf{n}$. Equations (2) and (3) can be further rewritten as

$$
\begin{gathered}
\hat{u}^{n}=\ln _{x}^{2} \varepsilon_{x x}+\ln _{y}^{2} \varepsilon_{y y}+2 \ln _{x} n_{y} \varepsilon_{x y} \\
\hat{u}^{s}=-\ln _{x} n_{y} \varepsilon_{x x}+\ln _{x} n_{y} \varepsilon_{y y}+l\left(n_{x}^{2}-n_{y}^{2}\right) \varepsilon_{x y}
\end{gathered}
$$

Using the matrix form as $\hat{\mathbf{u}}=\mathbf{T s}$ to represent these equations, where $\mathbf{T}$ and $\mathbf{s}$ are given as 


$$
\begin{gathered}
\mathbf{T}=l\left[\begin{array}{ccc}
n_{x}^{2} & n_{y}^{2} & 2 n_{x} n_{y} \\
-n_{x} n_{y} & n_{x} n_{y} & n_{x}^{2}-n_{y}^{2}
\end{array}\right] \\
\mathbf{s}=\left[\varepsilon_{x x}, \varepsilon_{y y}, \varepsilon_{x y}\right]^{\mathrm{T}}
\end{gathered}
$$

Here $\mathbf{s}$ is the vector composed of the three strain components. It can be calculated according to

$$
\mathbf{s}=\mathbf{B u}
$$

where $\mathbf{B}$ is the interpolation matrix and $\mathbf{u}$ is the displacement vector. For the type-I bond (see Fig. 1) which only belongs to one triangular element, we use the common finite element interpolation which gives

$$
\begin{gathered}
\mathbf{B}=\left[\begin{array}{cccccc}
N_{1, x}^{\mathrm{e}} & 0 & N_{2, x}^{\mathrm{e}} & 0 & N_{3, x}^{\mathrm{e}} & 0 \\
0 & N_{1, y}^{\mathrm{e}} & 0 & N_{2, y}^{\mathrm{e}} & 0 & N_{3, y}^{\mathrm{e}} \\
\frac{1}{2} N_{1, y}^{\mathrm{e}} & \frac{1}{2} N_{1, x}^{\mathrm{e}} & \frac{1}{2} N_{2, y}^{\mathrm{e}} & \frac{1}{2} N_{2, x}^{\mathrm{e}} & \frac{1}{2} N_{3, y}^{\mathrm{e}} & \frac{1}{2} N_{3, x}^{\mathrm{e}}
\end{array}\right] \\
\mathbf{u}=\left[u_{1}, v_{1}, u_{2}, v_{2}, u_{3}, v_{3}\right]^{\mathrm{T}}
\end{gathered}
$$

where $N_{i}^{\mathrm{e}}$ is the element shape function associated with the node $i$. For the type-II bond (see Fig. 1) which belongs to two triangular elements, a moving least squares (MLS) procedure [39] is adopted to calculate $\mathbf{s}$. The explicit computation of derivatives of the MLS shape functions is provided in [39]. The method will not involve any matrix inversion or linear system solving operations. Here, the explicit formularies of MLS shape functions of 4 nodes under linear basis provided in [39] will be directly used. Details of this explicit MLS computation method can be found in [39]. In this sense, we will have

$$
\begin{gathered}
\mathbf{B}=\left[\begin{array}{cccccccc}
N_{1, x} & 0 & N_{2, x} & 0 & N_{3, x} & 0 & N_{4, x} & 0 \\
0 & N_{1, y} & 0 & N_{2, y} & 0 & N_{3, y} & 0 & N_{4, y} \\
\frac{1}{2} N_{1, y} & \frac{1}{2} N_{1, x} & \frac{1}{2} N_{2, y} & \frac{1}{2} N_{2, x} & \frac{1}{2} N_{3, y} & \frac{1}{2} N_{3, x} & \frac{1}{2} N_{4, y} & \frac{1}{2} N_{4, x}
\end{array}\right] \\
\mathbf{u}=\left[u_{1}, v_{1}, u_{2}, v_{2}, u_{3}, v_{3}, u_{4}, v_{4}\right]^{\mathrm{T}}
\end{gathered}
$$

where $N_{i, x}$ and $N_{i, y}$ are the diffusive derivatives given by 


$$
\begin{aligned}
& N_{i, x}=\frac{w_{i}}{d} \sum_{j \neq i} \sum_{k>j, k \neq i} w_{j} w_{k}\left(y_{j}-y_{k}\right) \Theta\left(\mathbf{x}_{i}, \mathbf{x}_{j}, \mathbf{x}_{k}\right) \\
& N_{i, y}=\frac{w_{i}}{d} \sum_{j \neq i} \sum_{k>j, k \neq i} w_{j} w_{k}\left(x_{k}-x_{j}\right) \Theta\left(\mathbf{x}_{i}, \mathbf{x}_{j}, \mathbf{x}_{k}\right)
\end{aligned}
$$

with

$$
\begin{gathered}
\Theta\left(\mathbf{x}_{i}, \mathbf{x}_{j}, \mathbf{x}_{k}\right)=-x_{j} y_{i}+x_{k} y_{i}+x_{i} y_{j}-x_{k} y_{j}-x_{i} y_{k}+x_{j} y_{k} \\
d=\sum_{i=1,2} \sum_{j=i+1,3} \sum_{k=j+1,4} w_{i} w_{j} w_{k}\left(\Theta\left(\mathbf{x}_{i}, \mathbf{x}_{j}, \mathbf{x}_{k}\right)\right)^{2}
\end{gathered}
$$

The weight function $w$ used in this paper is the widely used Gauss function

$$
w(r)=e^{\left(-3 \hat{r}^{2}\right)}
$$

where $\hat{r}=r / r_{\max }$ with $r=\sqrt{\left(x_{\mathrm{m}}-x\right)^{2}+\left(y_{\mathrm{m}}-y\right)^{2}}$ and $\left(x_{\mathrm{m}}, y_{\mathrm{m}}\right)$ being the reference point (the center of the bond in this paper) and $r_{\max }$ is the maximum distance between the reference point and the nodes.

The strain energy stored in each bond is

$$
\Pi_{\mathrm{b}}=\frac{1}{2} \widehat{\mathbf{u}} K_{\text {bond }} \hat{\mathbf{u}}^{\mathrm{T}}
$$

The global stiffness matrix contributed by each bond is obtained as

$$
K^{\mathrm{b}}=\left[\frac{\partial^{2} \Pi_{\mathrm{b}}}{\partial u_{i} \partial u_{j}}\right]=(\mathbf{T B})^{T} \mathrm{~K}_{\text {bond }} \mathbf{T B}
$$

Finally, the global stiffness matrix is assembled bond by bond. The boundary conditions specified by displacement or force are treated in the same method as in the standard FEM. The proposed model with $\hat{\mathbf{u}}$ calculated from the local strain as described before is denoted as Distinct Lattice Spring Model (DLSM). A proof of rotationally invariant property of DLSM is given Appendix A. Hereafter, the proposed model with $\hat{\mathbf{u}}$ obtained directly from the particle displacements is called 
Classical Lattice Spring Model (CLSM) and the stiffness element of each bond can be simply written as

$$
\mathbf{K}^{\mathrm{b}}=\hat{\mathbf{T}}^{T} \mathbf{K}_{\text {bond }} \hat{\mathbf{T}}
$$

where $\hat{\mathbf{T}}$ is given as

$$
\hat{\mathbf{T}}=\left[\begin{array}{cccc}
n_{x} & n_{y} & -n_{x} & -n_{y} \\
-n_{y} & n_{x} & n_{y} & -n_{x}
\end{array}\right]
$$

\section{Relationship between spring parameters and elastic constants}

In this section, the relationship between spring parameters and elastic constants is derived by following the approach used in the virtual multi-dimensional internal bond model [40, 41]. The total strain energy stored per unit volume is

$$
\Pi=\frac{\sum \Pi_{\mathrm{b}}}{A \Delta}=\sum \frac{l^{2}\left(k_{\mathrm{n}} \xi_{i} \varepsilon_{i j} \xi_{j} \xi_{n} \varepsilon_{n m} \xi_{m}+k_{\mathrm{s}} \xi_{i} \varepsilon_{i j} \eta_{j} \xi_{n} \varepsilon_{n m} \eta_{m}\right)}{2 A \Delta}
$$

where $A$ is the area of the modeling domain and $\Delta$ is the unit length in the third dimension, $\xi$ is the direction vector of the bond which is $\left(n_{x}, n_{y}\right)$ and $\boldsymbol{\eta}$ is the unit vector perpendicular to $\xi$ which is $\left(-n_{y}, n_{x}\right)$. The stress tensor of the continuum can be obtained through the Cauchy-born rule [42] and the hyperelastic theory [43] and it can be written as

$$
\sigma_{i j}=\frac{\partial \Pi}{\partial \varepsilon_{i j}}=\sum \frac{l^{2}\left(k_{\mathrm{n}} \xi_{i} \xi_{j} \xi_{n} \varepsilon_{n m} \xi_{m}+k_{\mathrm{s}} \xi_{i} \eta_{j} \xi_{n} \varepsilon_{n m} \eta_{m}\right)}{A \Delta}
$$

The elastic modulus is expressed as

$$
c_{i j n m}=\frac{\partial^{2} \Pi}{\partial \varepsilon_{i j} \partial \varepsilon_{n m}}=\sum \frac{l^{2}\left(k_{\mathrm{n}} \xi_{i} \xi_{j} \xi_{n} \xi_{m}+k_{\mathrm{s}} \xi_{i} \eta_{j} \xi_{n} \eta_{m}\right)}{A \Delta}
$$


When the total number of bonds is large enough, Equation (22) can be written in the integral form as

$$
c_{i j n m}=\frac{1}{A \Delta} \int_{l_{1}}^{l_{2}} \int_{0}^{\pi} l^{2}\left(k_{\mathrm{n}} \xi_{i} \xi_{j} \xi_{n} \xi_{m}+k_{\mathrm{s}} \xi_{i} \eta_{j} \xi_{n} \eta_{m}\right) D(l, \beta) d \beta d l
$$

where $D(l, \beta) d \beta d l$ is the number of bonds with bond length between $(l, l+d l)$ and bond orientation between $(\beta, \beta+d \beta)$. For the isotropic material, the bonds distribute uniformly in each direction. So the bond distribution function $D(l, \beta)$ is reduced to $N(l) / \pi$ with $N(l) d l$ being the number of bonds with length between $(l, l+d l)$. In numerical methods e.g. FEM, the elastic tensor $c_{i j n m}$ is often written in the elastic matrix form as follows:

$$
\boldsymbol{\Omega}=\left[\begin{array}{lll}
C_{1111} & C_{1122} & \frac{1}{2}\left(C_{1112}+C_{1121}\right) \\
C_{2211} & C_{2222} & \frac{1}{2}\left(C_{2212}+C_{2221}\right) \\
C_{1211} & C_{1222} & \frac{1}{2}\left(C_{1212}+C_{1221}\right)
\end{array}\right]
$$

For the linear elastic cases, the tangent modulus is equal to the secant modulus and Equation (23) can be considered as the secant modulus. So the following relationship exists:

$$
\boldsymbol{\sigma}=\boldsymbol{\Omega} \cdot \boldsymbol{\varepsilon}
$$

where $\boldsymbol{\sigma}=\left[\sigma_{11}, \sigma_{22}, \sigma_{12}\right]^{\mathrm{T}}, \boldsymbol{\varepsilon}=\left[\varepsilon_{11}, \varepsilon_{22}, 2 \varepsilon_{12}\right]^{\mathrm{T}}$. Here $\sigma_{i j}$ and $\varepsilon_{i j}$ are the components of stress and strain tensor, respectively. By integrating Equation (23) and using Equation (24), the corresponding elastic matrix is obtained as:

$$
\boldsymbol{\Omega}=\frac{\int_{l_{1}}^{l_{2}} l^{2} N(l) d l}{8 A \Delta}\left[\begin{array}{ccc}
3 k_{\mathrm{n}}+k_{\mathrm{s}} & k_{\mathrm{n}}-k_{\mathrm{s}} & 0 \\
& 3 k_{\mathrm{n}}+k_{\mathrm{s}} & 0 \\
\text { symmetry } & & k_{\mathrm{n}}+k_{\mathrm{s}}
\end{array}\right]
$$

Let $\alpha^{2 D}=\int_{l_{1}}^{l_{2}} l^{2} N(l) d l / A \Delta$, then the relationship between the spring parameters $k_{\mathrm{n}}, k_{\mathrm{s}}$ and the macro elastic constants, i.e. the Young's modulus $E$ and the Poisson ratio $v$ can be obtained from Equation (26) as follows: 


$$
\begin{aligned}
& k_{\mathrm{n}}=\frac{2 E}{\alpha^{2 D}(1-v)} \\
& k_{\mathrm{s}}=\frac{2(1-3 v) E}{\alpha^{2 D}\left(1-v^{2}\right)}
\end{aligned}
$$

for the plane-stress problems and

$$
\begin{aligned}
& k_{\mathrm{n}}=\frac{2 E}{\alpha^{2 D}(1+v)(1-2 v)} \\
& k_{\mathrm{s}}=\frac{2(1-4 v) E}{\alpha^{2 D}(1+v)(1-2 v)}
\end{aligned}
$$

for the plain-strain problems. Given the geometry data of the lattice spring model, $\alpha^{2 D}$ can be estimated through:

$$
\alpha^{2 D}=\frac{\sum l_{i}^{2}}{A \Delta}
$$

where $l_{i}$ is the original length of the $i$ th bond. Equations (27) and (28) are used to estimate the two spring stiffnesses of the proposed LSM for numerical simulation of elastic problems. It is seen that the addition of shear spring allows the materials of Poisson's ratios less than $1 / 3$ (in case of plane stress) or $1 / 4$ (in case of plain strain) to be modeled. In the next section, numerical examples of validation and application of the proposed model will be presented.

\section{Numerical Examples}

\subsection{Beam subjected to bending}

The geometry and boundary conditions of this plain-stress problem are described in Figure 2 . The left side of the beam is fixed in the $\mathrm{x}$ direction and the left-bottom corner is fixed in both $\mathrm{x}$ and $\mathrm{y}$ directions. A shear stress ( $1 \mathrm{MPa})$ is applied on the right side of the beam. The top and bottom boundaries are subject to the stress free condition. The elastic constants of the material and the 
corresponding spring parameters for the proposed LSM are given in Table 1. The final algebraic equation assembled from the bond stiffness matrix given by Eq. (17) with the implementation of the boundary conditions is solved by a direct method for sparse matrix. The lattice size is $4 \mathrm{~m}$, which corresponds to a total of 1250 lattice nodes approximately. Figure 3 shows the displacement results predicted by FEM, CLSM and DLSM with the Poisson's ratio of 0.1 . The results of DLSM are in good agreement with those obtained by FEM, while this is not true for the results of CLSM. Compared to the displacement results obtained by FEM, the maximal relative errors of CLSM and DLSM (denoted as Err_1 and Err_2) are given in Table 1 for four different values of Poisson's ratio. In all cases the errors of DLSM are rather small, while the errors of CLSM are very large except for the case of Poisson's ratio equal to $1 / 3$ where shear spring is absent. Therefore, it can be concluded that shear spring must be introduced by preserving rotational invariance as done in DLSM in order to reproduce reasonably the elastic solutions for Poisson's ratios other than 1/3.

The convergence of DLSM is studied by solving the same problem with different lattice sizes. Figure 4 shows that when lattice size becomes smaller and smaller, the result of DLSM gets closer and closer to the reference one obtained by FEM with a fine mesh. The effect of lattice structure is also studied by comparing the results of four different lattice structures as shown in Figure 5, in which structure $a$ is made of particles with a slight irregular distribution, structure $b$ consists of particles with a regular distribution, and structures $c$ and $d$ are obtained by randomly moving the particles in structures $a$ and $b$ respectively. The model parameters and the results for this study are summarized in Table 2, from which it is observed that the random lattice structure gives better results than the regular lattice does. The reason is that the relationship between the model 
parameters and the material constants is derived based on the assumption that the bond orientation distribution is uniform. Hence, a random lattice is preferable when applying the relationship to obtain the model parameters from the material constants.

By inspection of equations (27) and (28), it appears that the proposed model still face a theoretical limitation, i.e., Poisson's ratios greater than $1 / 3$ (plane stress) or $1 / 4$ (plane strain) cannot be modeled. However, this limitation is not true practically for DLSM, as the shear spring of negative stiffness can be added in the model and the final algebraic equation is still solvable. The behavior of the model with negative shear springs shall be examined in the next example.

\subsection{Square hole subjected to compression}

In this subsection, a more complex plain-stress problem is solved by DLSM. Figure 6 shows the geometry, the loading condition, and the lattice structure for this problem. The elastic constants are $E=10^{3} \mathrm{MPa}$ and $v=0.2$ or 0.4 . The spring parameters are obtained according to Eq. (27). The results are presented in figures 7 and 8 . Again, a good match between the results by DLSM and the elastic solutions by FEM is observed. It is important to point out that when $v=0.4$ the shear stiffness is negative according to Eq. (27). However, the final algebraic equation can be solved and yields the correct results (see Fig. 8). Of course, like FEM, the proposed method cannot solve the case of $v=0.5$. However, this is not a deficiency of the method, because incompressible solid materials do not exist. The negative stiffness of shear spring seems non-physical, but the negative stiffness can have a physical explanation molecular level. This is discussed in Appendix B. Moreover, in reality materials with negative stiffness are also reported and used for extreme 
damping in composite materials [44]. In this sense, DLSM provide a solution for the Poisson's ratio problem existing in the lattice spring models.

\subsection{Fracture simulation}

The proposed model is applied to the fracture simulation of a solid specimen with a side notch subjected to quasi-static tensile loading in the plain-stress condition. The geometry and the loading setup are shown in Figure 9. The controlled displacement on the top is $0.01 \mathrm{~m}$. The elastic constants of the material are $E=10^{3} \mathrm{MPa}$ and $v=0.2$. Again, the spring parameters are obtained according to Eq. (27). Because the purpose of this example is to demonstrate the easy feature of the model for fracture simulation, we only consider the tensile failure of bond, which occurs when

$$
-F_{n}>F_{t}
$$

where $F_{n}$ is the normal force of the bond and $F_{t}=\gamma_{t} k_{n}$ is the tensile strength of the bond with $\gamma_{t}$ being the limit value of the bond's stretching. More sophisticated bond fracture criteria can be implemented in the model for more realistic modelling of fracture processes. During the simulation, the normal deformation of each bond is calculated using Eq. (5), from which the normal force is obtained by multiplying the normal stiffness. Then, according to Eq. (30), the status of each bond (failure or not) is obtained. Whenever a bond fails, it is deleted from the calculation procedure. The simulation was performed using $\gamma_{t}=0.0003$. The damage pattern is presented in Figure 10 for four stages. The bond in which failure occurs is marked by double red lines around the center of the bond. With regard to the crack patterns obtained, the simulation gives a realistic description of the fracture process of the notched solid specimen under tensile loading. 


\section{Conclusions}

The paper presents an alternative MLS-based lattice spring model, in which the deformation of shear springs is calculated by using the local strain instead of the particle displacements. With this technique, the proposed model can represent the diversity of Poisson's ratio while preserving rotational invariance. Based on the Cauchy-born rules, the relationship which bridges the spring parameters and the elastic constants is derived. The model is capable of capturing the mechanical behavior of an elastic solid as demonstrated numerically. It is found that rotational invariance should be respected in order to have good agreements with corresponding elastic solutions by FEM. Negative shear stiffness does not present numerical problems and can be adopted in the proposed model. With this approach, the full range of Poisson's ratio of an elastic solid can be modeled. The fracture process of a notched specimen under tensile loading is simulated by the model. The results show that the proposed lattice spring model is suitable and convenient for fracture modeling. The proposed method is based on the discrete spring model and has no integration involved in the calculation procedure. Therefore, it has the potential of being fully meshless and more advantageous than FEM in modeling dynamic fracturing problems. The further development of fully meshless explicit 3D DLSM and more realistic modeling of fracture using advanced failure criteria will be reported in the forthcoming papers. 


\section{Appendix A}

\section{Proof of rotation invariant in DLSM}

In this appendix, Equation (3) used for evaluating the deformation of shear springs in DLSM is derived. First, consider a cubic unit (a microelement of the elasticity body) containing a bond connecting two particles as shown in Figure A1.

The complete $1^{\text {st }}$ order displacement function of the cubic is

$$
\left(\begin{array}{l}
u_{x} \\
u_{y}
\end{array}\right)=\left(\begin{array}{lll}
a_{0} & a_{1} & a_{2} \\
b_{0} & b_{1} & b_{2}
\end{array}\right)\left(\begin{array}{l}
1 \\
x \\
y
\end{array}\right)
$$

Assuming the center of the block at $\left(x_{c}, y_{c}\right)$, then its displacement is represented by

$$
\left(\begin{array}{l}
u_{c x} \\
u_{c y}
\end{array}\right)=\left(\begin{array}{lll}
a_{0} & a_{1} & a_{2} \\
b_{0} & b_{1} & b_{2}
\end{array}\right)\left(\begin{array}{c}
1 \\
x_{c} \\
y_{c}
\end{array}\right)
$$

Subtracting (A.2) from (A.1) gives

$$
\left(\begin{array}{l}
u_{x} \\
u_{y}
\end{array}\right)-\left(\begin{array}{l}
u_{c x} \\
u_{c y}
\end{array}\right)=\left(\begin{array}{lll}
a_{0} & a_{1} & a_{2} \\
b_{0} & b_{1} & b_{2}
\end{array}\right)\left(\begin{array}{l}
1 \\
x \\
y
\end{array}\right)-\left(\begin{array}{ccc}
a_{0} & a_{1} & a_{2} \\
b_{0} & b_{1} & b_{2}
\end{array}\right)\left(\begin{array}{c}
1 \\
x_{c} \\
y_{c}
\end{array}\right)
$$

Equation (A.3) can be further written as

$$
\left(\begin{array}{l}
u_{x} \\
u_{y}
\end{array}\right)=\left(\begin{array}{l}
u_{c x} \\
u_{c y}
\end{array}\right)+\left(\begin{array}{cc}
a_{1} & 0 \\
0 & b_{2}
\end{array}\right)\left(\begin{array}{l}
x-x_{c} \\
y-y_{c}
\end{array}\right)+\left(\begin{array}{cc}
0 & a_{2} \\
b_{1} & 0
\end{array}\right)\left(\begin{array}{l}
x-x_{c} \\
y-y_{c}
\end{array}\right)
$$

From (A.4), we have

$$
\begin{gathered}
\varepsilon_{x x}=\frac{\partial u_{x}}{\partial x}=a_{1} \\
\varepsilon_{y y}=\frac{\partial u_{y}}{\partial y}=b_{2}
\end{gathered}
$$




$$
\begin{gathered}
\varepsilon_{x y}=\frac{1}{2}\left(\frac{\partial u_{y}}{\partial x}+\frac{\partial u_{x}}{\partial y}\right)=\frac{1}{2}\left(b_{1}+a_{2}\right) \\
\omega=\frac{1}{2}\left(\frac{\partial u_{y}}{\partial x}-\frac{\partial u_{x}}{\partial y}\right)=\frac{1}{2}\left(b_{1}-a_{2}\right)
\end{gathered}
$$

Using the above relations, Equation (A.4) can be transformed into

$$
\left(\begin{array}{l}
u_{x} \\
u_{y}
\end{array}\right)=\left[\mathrm{D}_{0}\right]\left(\begin{array}{l}
u_{c x} \\
u_{c y}
\end{array}\right)+\left[\mathrm{D}_{1}\right](\omega)+\left[\mathrm{D}_{2}\right]\left(\begin{array}{l}
\varepsilon_{x x} \\
\varepsilon_{y y}
\end{array}\right)+\left[\mathrm{D}_{3}\right]\left(\varepsilon_{x y}\right)
$$

where

$$
\begin{gathered}
{\left[\mathrm{D}_{0}\right]=\left(\begin{array}{ll}
1 & 0 \\
0 & 1
\end{array}\right)} \\
{\left[\mathrm{D}_{1}\right]=\left(\begin{array}{c}
-\left(y-y_{c}\right) \\
x-x_{c}
\end{array}\right)} \\
{\left[\mathrm{D}_{2}\right]=\left(\begin{array}{cc}
x-x_{c} & 0 \\
0 & y-y_{c}
\end{array}\right)} \\
{\left[\mathrm{D}_{3}\right]=\left(\begin{array}{c}
y-y_{c} \\
x-x_{c}
\end{array}\right)}
\end{gathered}
$$

Denoting the coordinates of the two particles in the cubic as $\left(x_{1}, y_{1}\right)$ and $\left(x_{2}, y_{2}\right)$ and their displacement as $\left(u_{1}, v_{1}\right)$ and $\left(u_{2}, v_{2}\right)$, the relative displacement vector between the two particles is

$$
\left(\begin{array}{l}
u_{12 x} \\
u_{12 y}
\end{array}\right)=\left(\begin{array}{l}
u_{2 x}-u_{1 x} \\
u_{2 y}-u_{1 y}
\end{array}\right)
$$

and the normal unit vector is

$$
\left(\begin{array}{l}
n_{x} \\
n_{y}
\end{array}\right)=\left(\begin{array}{l}
\frac{x_{2}-x_{1}}{l} \\
\frac{y_{2}-y_{1}}{l}
\end{array}\right)
$$

where $l$ is the length of the bond. The relative normal displacement vector is defined as 


$$
\left(\begin{array}{l}
u_{12 x}^{n} \\
u_{12 y}^{n}
\end{array}\right)=\left(\left(\begin{array}{l}
u_{12 x} \\
u_{12 y}
\end{array}\right)^{T}\left(\begin{array}{l}
n_{x} \\
n_{y}
\end{array}\right)\right)\left(\begin{array}{l}
n_{x} \\
n_{y}
\end{array}\right)
$$

By vector operation, the relative shear displacement vector is obtained as

$$
\left(\begin{array}{l}
u_{12 x}^{s} \\
u_{12 y}^{s}
\end{array}\right)=\left(\begin{array}{l}
u_{12 x} \\
u_{12 y}
\end{array}\right)-\left(\begin{array}{l}
u_{12 x}^{n} \\
u_{12 y}^{n}
\end{array}\right)=\left(\begin{array}{l}
u_{12 x} \\
u_{12 y}
\end{array}\right)-\left(\left(\begin{array}{l}
u_{12 x} \\
u_{12 y}
\end{array}\right)^{T}\left(\begin{array}{l}
n_{x} \\
n_{y}
\end{array}\right)\right)\left(\begin{array}{l}
n_{x} \\
n_{y}
\end{array}\right)
$$

Now, by applying Equation (A.5), the relative displacement vector can be represented as

$$
\left(\begin{array}{c}
u_{2 x}-u_{1 x} \\
u_{2 y}-u_{1 y}
\end{array}\right)=\underbrace{\left(\begin{array}{cc}
x_{2}-x_{1} & 0 \\
0 & y_{2}-y_{1}
\end{array}\right)\left(\begin{array}{c}
\varepsilon_{x x} \\
\varepsilon_{y y}
\end{array}\right)+\varepsilon_{x y}\left(\begin{array}{c}
y_{2}-y_{1} \\
x_{2}-x_{1}
\end{array}\right)}_{\text {strain related term }}+\underbrace{\omega\left(\begin{array}{c}
-\left(y_{2}-y_{1}\right) \\
x_{2}-x_{1}
\end{array}\right)}_{\text {rotation related term }}
$$

With the above equation, it is straightforward to show that the relative normal displacement vector is only dependent on the strain related term because of the following equivalence

$$
\left(\begin{array}{c}
-\left(y_{2}-y_{1}\right) \\
x_{2}-x_{1}
\end{array}\right)^{T}\left(\begin{array}{c}
x_{2}-x_{1} \\
y_{2}-y_{1}
\end{array}\right)=0
$$

However, for the relative shear displacement vector, if we directly substitute (A.10) into (A.9), the rotation related term will not vanish. It is known that rigid rotation of the cubic should not produce strain energy. Therefore, in DLSM, the rotation related term is removed from the calculation of the relative shear displacement vector, namely, the relative displacement vector in (A.9) is not calculated anymore by using (A.10) or (A.6), but by using the following

$$
\left(\begin{array}{l}
\hat{u}_{12 x} \\
\hat{u}_{12 y}
\end{array}\right)=\left(\begin{array}{cc}
x_{2}-x_{1} & 0 \\
0 & y_{2}-y_{1}
\end{array}\right)\left(\begin{array}{l}
\varepsilon_{x x} \\
\varepsilon_{y y}
\end{array}\right)+\varepsilon_{x y}\left(\begin{array}{l}
y_{2}-y_{1} \\
x_{2}-x_{1}
\end{array}\right)=\left(\begin{array}{ll}
\varepsilon_{x x} & \varepsilon_{x y} \\
\varepsilon_{x y} & \varepsilon_{y y}
\end{array}\right)\left(\begin{array}{l}
x_{2}-x_{1} \\
y_{2}-y_{1}
\end{array}\right)
$$

Writing (A.11) in the vector form, we get

$$
\hat{\mathbf{u}}_{i j}=[\boldsymbol{\varepsilon}] \cdot \mathbf{n} l
$$

Finally, the relative shear displacement vector (the vector form of (A.9)) can be written as

$$
\hat{\mathbf{u}}_{i j}^{s}=[\boldsymbol{\varepsilon}] \cdot \mathbf{n} l-(([\boldsymbol{\varepsilon}] \cdot \mathbf{n} l) \cdot \mathbf{n}) \mathbf{n}
$$


Then, the magnitude of shear deformation can be given as

$$
\hat{u}^{s}=([\boldsymbol{\varepsilon}] \cdot \mathbf{n} l-(([\boldsymbol{\varepsilon}] \cdot \mathbf{n} l) \cdot \mathbf{n}) \mathbf{n}) \cdot \mathbf{n}^{\prime}
$$

where $\mathbf{n}^{\prime}=\left(-n_{y}, n_{x}\right)^{T}$ is the unit vector perpendicular to $\mathbf{n}$.

Moreover, consider one rigid body rotation defined by

$$
\mathbf{u}(\mathbf{x})=\omega \times \mathbf{x}
$$

where $\omega$ is the angular displacement vector with components $\left[\omega_{x}, \omega_{y}, \omega_{z}\right]^{\mathrm{T}}$. By simple derivation, the true gradient of this displacement field is found to be

$$
\nabla \mathbf{u}=\left(\begin{array}{ccc}
0 & -\omega_{z} & \omega_{y} \\
\omega_{z} & 0 & -\omega_{x} \\
-\omega_{y} & \omega_{x} & 0
\end{array}\right)
$$

It is obvious that the strain tensor $\boldsymbol{\varepsilon}=\left(\nabla \mathbf{u}+\nabla \mathbf{u}^{\mathrm{T}}\right) / 2$ vanishes given the skew nature of $\nabla \mathbf{u}$. The least square approximation adopted in DLSM to calculate the gradient of the displacement field is first-order consistent, i.e., it is able to reproduce any linear function and its gradient, so the correct skew nature of $\nabla \mathbf{u}$ is kept numerically. Therefore the calculated strain $\boldsymbol{\varepsilon}$ is also invariant with respect to the rigid body rotation.

Overall, it is ensured that the DLSM model is rotationally invariant in the sense that the strain energy is independent of rigid rotation. 


\section{Appendix B}

\section{Physical interpretation of negative spring}

Given a molecular model as shown in Figure B1(a), $\Phi_{B}\left(u_{s}\right)$ is the potential variation at molecular $\mathrm{B}$ versus the displacement of molecular $\mathrm{A}$ in the shear direction between A-B. The shape of $\Phi_{B}\left(u_{s}\right)$ determines the shear stiffness (see Figure B1(b)). When the potential function is a constant, the shear stiffness equals to zero as there is no work needs to do for a displacement. The shear stiffness is positive/negative when the potential function is of an upward/downward bowl shape. Therefore, the proof of negative shear spring can be based on the potential functions used in MD simulation. It is known that the Poisson's ratio of silver is 0.37 , which corresponds to negative shear spring stiffness in the DLSM model. The atomic lattice structure of sliver is shown in Figure B2. The Finnis-Sinclair potential proposed by Sutton and Chen [45] can be used to describe silver, which can be written as

$$
P_{i}=\varepsilon\left(\sum_{\substack{j=1 \\ j \neq i}}^{N}\left(\frac{\sigma}{r_{i j}}\right)^{n}-c \sqrt{\sum_{\substack{j=1 \\ j \neq i}}^{N}\left(\frac{\sigma}{r_{i j}}\right)^{m}}\right)
$$

Both repulsive and attractive contributions are included in this potential. The repulsive part is realized by a pair potential, while the attractive part is realized by a many-body potential. The parameters $n, m, \varepsilon, \sigma$ and $c$ are material dependent and related to the specific lattice type. The parameter values for silver are given in Table B1.

The potential variation on atom $\mathrm{B}$ due to the movement of atom $\mathrm{A}$ in different shear planes (see Figure B2) can be calculated based on Equation (B.1) and the lattice structure information. The results for silver are shown in Figure B3. It can be seen that the shape of the variation function is 
exactly of the downward bowl shape, which indicates that the shear stiffness is negative. This is consistent with the fact that the Poisson's ratio of silver is greater than the critical value $(0.25$ for $3 \mathrm{D}$ and 0.33 for $2 \mathrm{D})$. 


\section{REFERENCES}

1. Ostoja-Starzewski M, Sheng PY, Jasiuk I. Damage patterns and constitutive response of random matrix-inclusion composites. Eng Fract Mech 1997; 58(5-6):581-606.

2. Buxton GA, Care CM, Cleaver DJ. A lattice spring model of heterogeneous materials with plasticity. Modell Simul Mater Sci Eng 2001; 9(6):485-97.

3. Hrennikoff A. Solution of problems of elasticity by the framework method. ASME J. Appl. Mech. 1941; 8:A619-A715.

4. Lemieux MA, Breton P, Tremblay AMS. Unified approach to numerical transfer matrix methods for disordered systems: applications to mixed crystals and to elasticity percolation. J. Physique Lett. 1985; 46:1-7.

5. Ray P, Chakrabarti BK. A microscopic approach to the statistical fracture analysis of disordered brittle solids. Solid State Commun. 1985; 53(5):477-479.

6. Sahimi M, Goddard JD. Elastic percolation models for cohesive mechanical failure in heterogeneous systems. Phys. Rev. B 1986; 33:7848-7851.

7. de Arcangelis L, Hansen A, Herrmann HJ, Roux S. Scaling laws in fracture. Phys. Rev. B 1989; 40:877-880.

8. Beale PD, Srolovitz DJ. Elastic fracture in random materials. Phys. Rev. B 1988; 37(10):5500-7.

9. Srolovitz DJ, Beale PD. Computer simulation of failure in an elastic model with randomly distributed defects. J. Am. Ceram. Soc. 1988; 71(5):362-9.

10.Heermann HJ, Kertész J, De Arcangelis L. Fractal shapes of deterministic cracks. Europhys. Lett. $1989 ; 10: 147-152$.

11. Termonia Y, Meakin P. Formation of fractal cracks in a kinetic fracture model. Nature 1986; 
320(6061):429-431.

12. Meakin P. Stress distribution for a rigid fractal embedded in a two-dimensional elastic medium. Phys. Rev. A 1987; 36:325-331.

13. Meakin P. A simple model for elastic fracture in thin films. Thin Solid Films 1987; 151:165-190.

14. Curtin WA, Scher H. Mechanics modeling using a spring network. J. Mater. Res. 1990; $5: 554-562$.

15. Blumberg-Selinger RL, Wang ZC, Gelbart WM, Benshaul A. Statistical-thermodynamic approach to fracture. Phys. Rev. A 1991; 43:4396-4400.

16. Hassold GN, Srolovitz DJ. Brittle fracture in materials with random defects. Phys. Rev. B 1989; 39(13):9273-81.

17. Caldarelli G, Castellano C, Petri A. Criticality in models for fracture in disordered media. Physica A: Statistical Mechanics and its Applications 1999; 270(1):15-20.

18. Parisi A, Caldarelli G. Self-affine properties of fractures in brittle materials. Physica A: Statistical Mechanics and its Applications 2000; 280(1):161-5.

19. Ostoja-Starzewski M, Sheng PY, Alzebdeh K. Spring network models in elasticity and fracture of composites and polycrystals. Computational Materials Science 1996; 7(1-2):82-93.

20. Schwartz LM, Feng S, Thorpe MF, Sen PN. Behavior of depleted elastic networks: Comparison of effective-medium and numerical calculations. Phys. Rev. B 1985; 32(7):4607-17.

21. Sahimi M, Arbabi S. Percolation and fracture in disordered solids and granular media: Approach to a fixed point. Phys Rev Lett 1992; 68(5):608-11.

22. Monette L, Anderson MP. Elastic and fracture properties of the two-dimensional triangular and square lattices. Modell Simul Mater Sci Eng 1994; 2(1):53-66. 
23. Lilliu G, van Mier JGM. 3D lattice type fracture model for concrete. Eng. Fract. Mech. 2003; 70:927-941.

24. Schlangen E, Garboczi EJ. Fracture simulations of concrete using lattice models: computational aspects. Eng. Fract. Mech. 1997; 57:319-332.

25. Karihaloo BL, Shao PF, Xiao QZ. Lattice modelling of the failure of particle composites. Eng. Fract. Mech. 2003; 70(17):2385-2406.

26. Liu JX, Deng SC, Zhang J, Liang NG. Lattice type of fracture model for concrete. Theor. Appl. Fract. Mech. 2007; 48(3):269-284.

27.Cundall PA, Strack ODL. Discrete numerical model for granular assemblies. Geotechnique 1979; 29(1):47-65.

28. Kawai T. New discrete models and their application to seismic response analysis of structures. Nucl Eng Des 1978; 48(1):207-229.

29. Zubelewicz A, Bažant, ZP. Interface element modeling of fracture in aggregate composites. $J$ Eng Mech 1987; 113(11):1619-1630.

30. Cusatis G, Bažant ZP, Cedolin L. Confinement-shear lattice model for concrete damage in tension and compression: I. Theory. J Eng Mech 2003; 129(12):1439-1448.

31. Potyondy DO, Cundall PA. A bonded-particle model for rock. Int J Rock Mech Min Sci 2004, 41(8):1329-1364.

32. Mustoe GGW. Generalized formulation of the discrete element method. Engineering Computations 1992; 9(2):181-190.

33. Kozicki J, Tejchman J. Modelling of fracture process in concrete using a novel lattice model. Granular Matter 2008; 10(5):377-388. 
34. Wang G, Al-Ostaz A, Cheng AHD, Mantena PR. Hybrid lattice particle modeling: Theoretical considerations for a 2D elastic spring network for dynamic fracture simulations. Computational Materials Science 2009; 44(4):1126-1134.

35. Ashurst WT, Hoover WG. Microscopic fracture studies in the two-dimensional triangular lattice. Phys Rev B 1976; 14(4):1465-1473.

36. Gusev AA. Finite element mapping for spring network representations of the mechanics of solids. Phys Rev Lett 2004; 93(3):034302-1.

37. Ostoja-Starzewski M. Lattice models in micromechanics. Appl. Mech. Rev. 2002; 55(1):35-59.

38. Ostoja-Starzewski M. Microstructural randomness and scaling in mechanics of materials, Chapman \& Hall/CRC/Taylor \& Francis, 2007.

39. Breitkopf P, Rassineux A, Touzot G, Villon P. Explicit form and efficient computation of MLS shape functions and their derivatives. Int J Numer Methods Eng 2000; 48(3):451-66.

40. Zhang ZN, Ge XR. Micromechanical consideration of tensile crack behavior based on virtual internal bond in contrast to cohesive stress. Theor Appl Fract Mech 2005; 43(3):342-59.

41. Zhao GF, Fang J, Zhao J. A new microstructure-based constitutive model for failure modeling of elastic continuum. European Journal of Mechanics, A/Solids 2009; submitted.

42. Tadmor EB, Ortiz M, Phillips R. Quasicontinuum analysis of defects in solids. Philosophical Magazine A 1996; 73(6):1529-93.

43. Marsden JE, Hughes TJR. Mathematical Foundations of Elasticity. Prentice-Hall: Englewood Cliffs, New Jersey, 1983.

44. Lakes RS, Lee T, Bersie A, Wang YC. Extreme damping in composite materials with negative-stiffness inclusions. Nature 2001; 410:565-567. 
45. Sutton A, Chen J. Long-range Finnis-Sinclair potentials. Philosophical Magazine Letters 1990;61: $139-146$. 


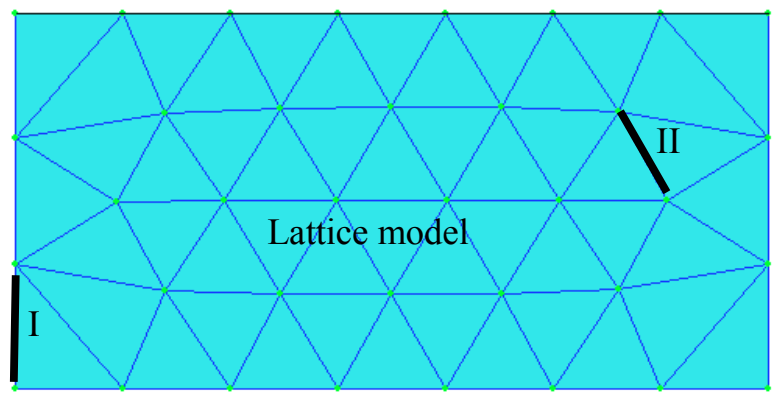

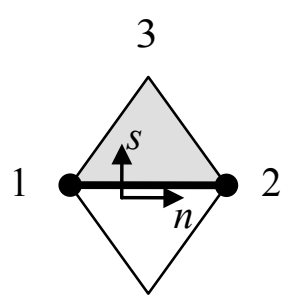

$4($ null $)$

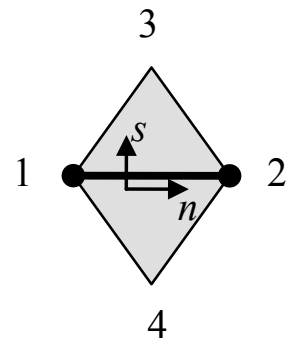

Type II

Figure 1. The 2D lattice spring model and the two types of bond. 


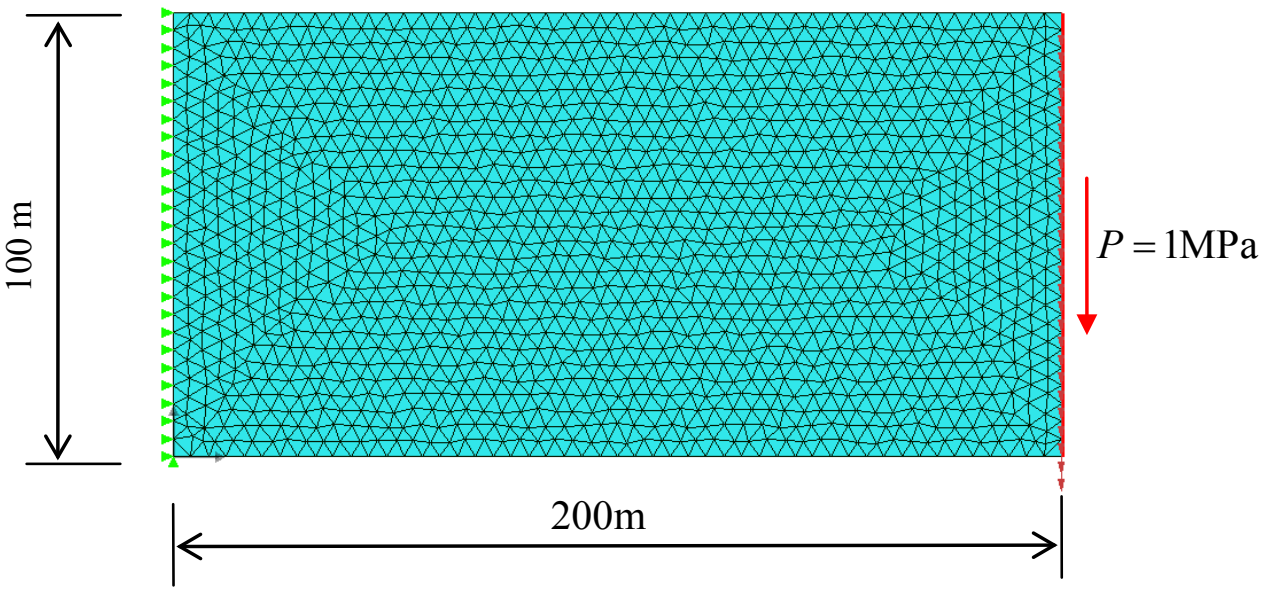

Figure 2. The geometry and boundary conditions for the beam bending problem. 


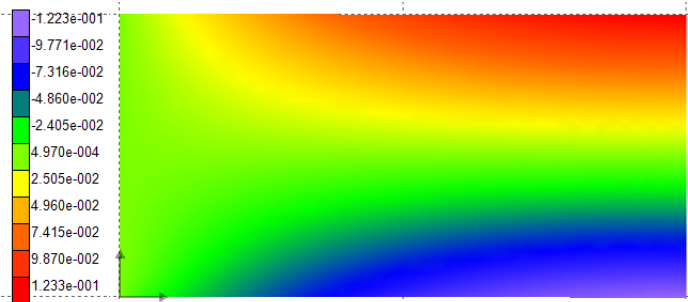

(a) The $\mathrm{x}$-direction displacement (FEM)

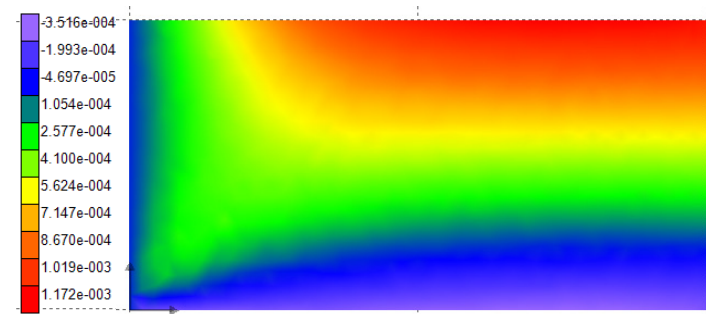

(c) The x-direction displacement (CLSM)

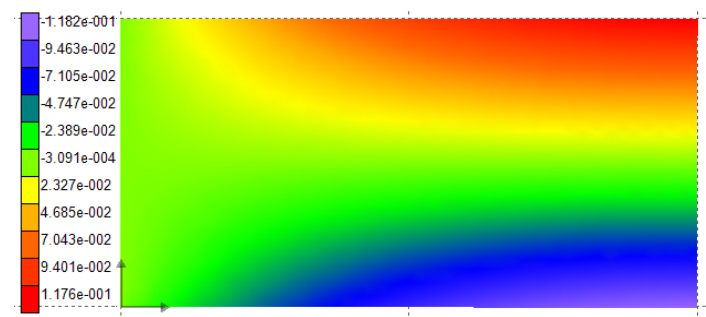

(e) The x-direction displacement (DLSM)

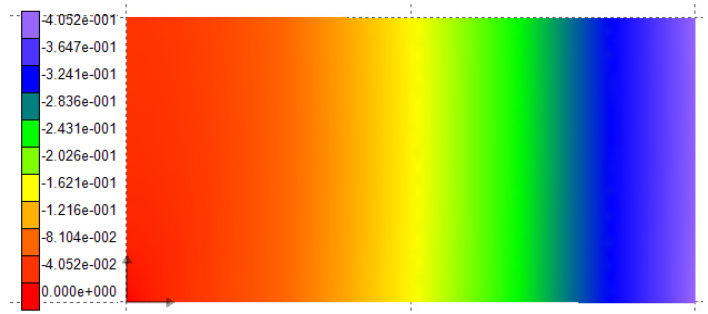

(b) The y-direction displacement (FEM)

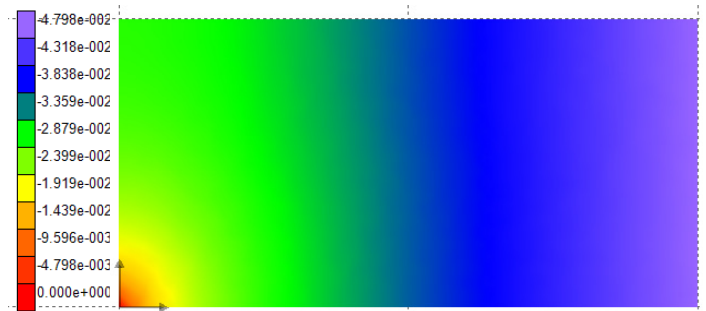

(d) The y-direction displacement (CLSM)

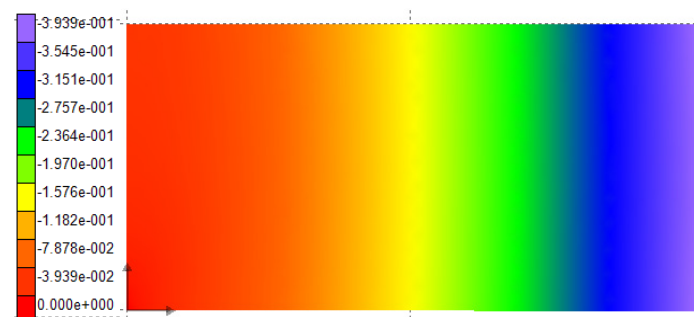

(f) The y-direction displacement (DLSM)

Figure 3. Contour plot of the displacement results predicted by FEM, CLSM and DLSM for the beam bending problem with $E=10^{4} \mathrm{Mpa}$ and $v=0.1$. 


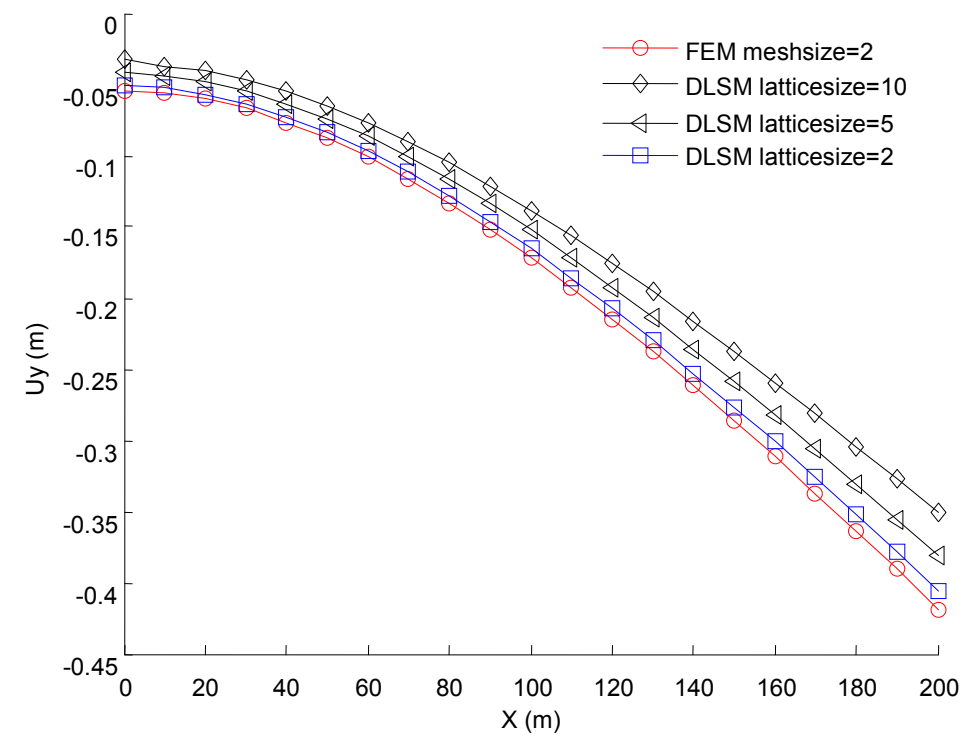

Figure 4. The y-direction displacement along the top surface predicted by DLSM with different lattice sizes for the beam bending problem with $E=10^{4} \mathrm{MPa}$ and $v=0.2$. 


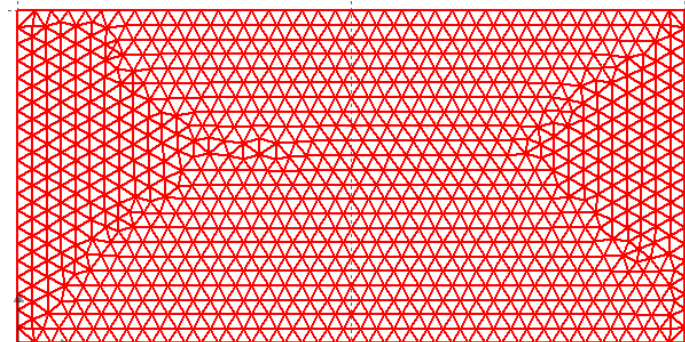

(a)

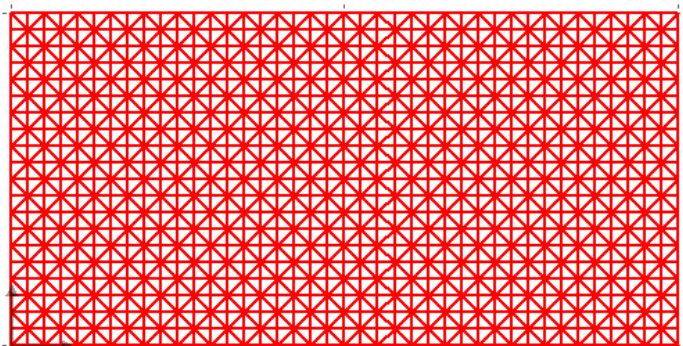

(b)

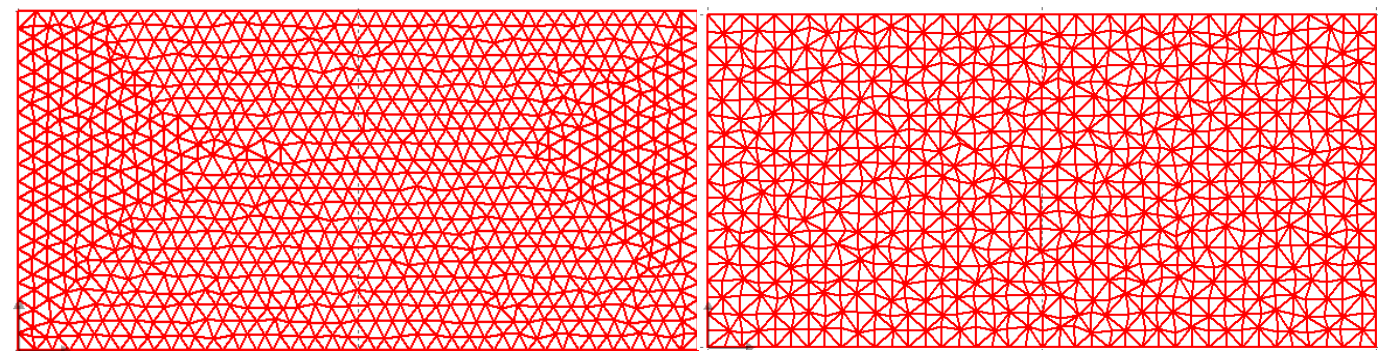

(c)

(d)

Figure 5. Different lattice structures for the beam bending problem. 


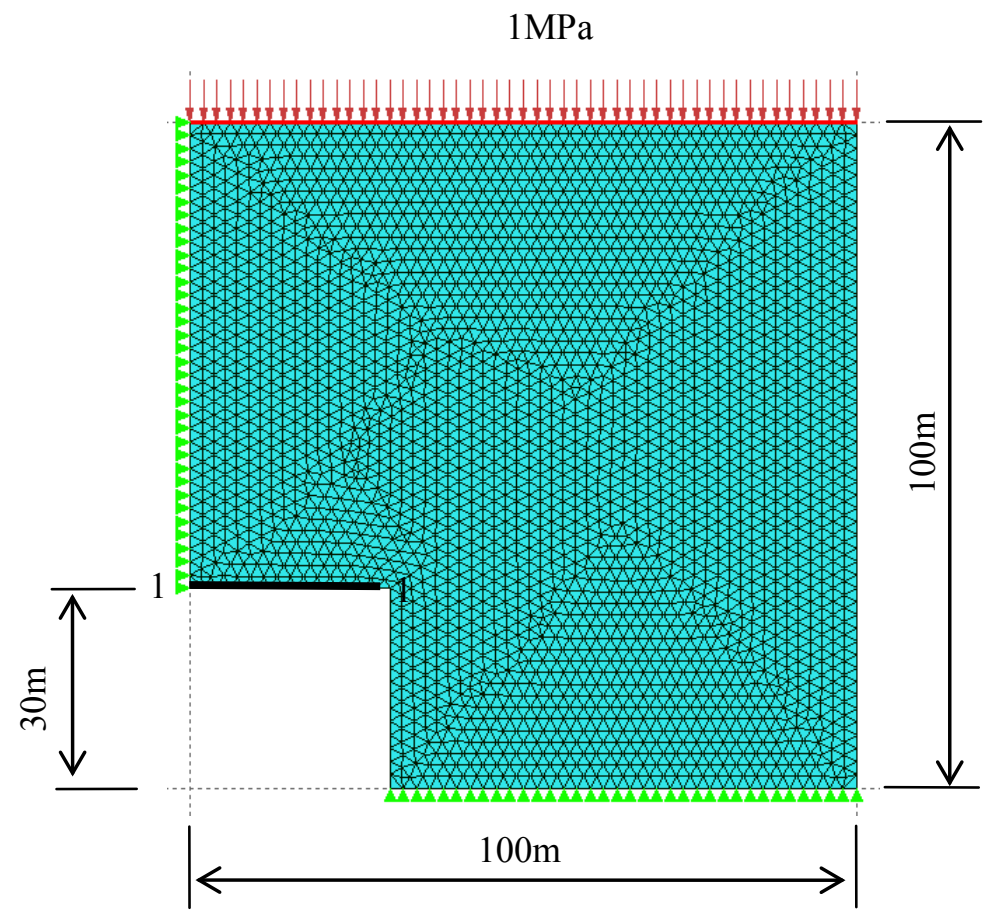

Figure 6 . The geometry and boundary conditions for the square hole problem. 

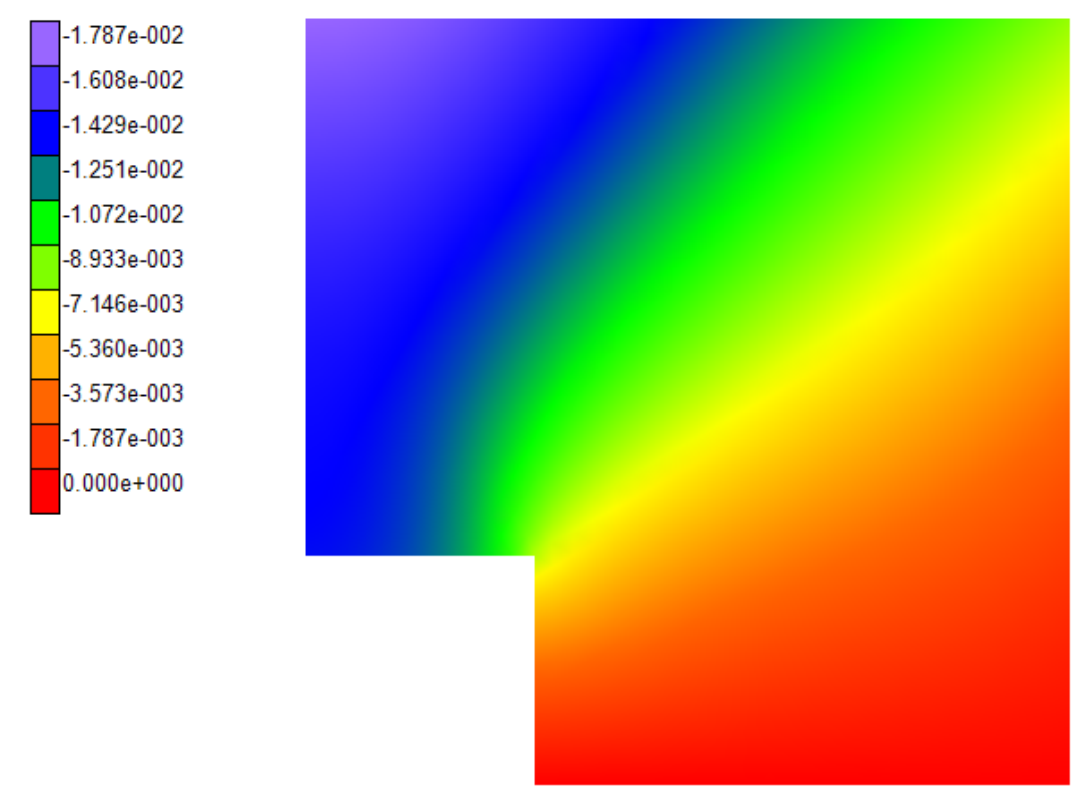

(a) FEM

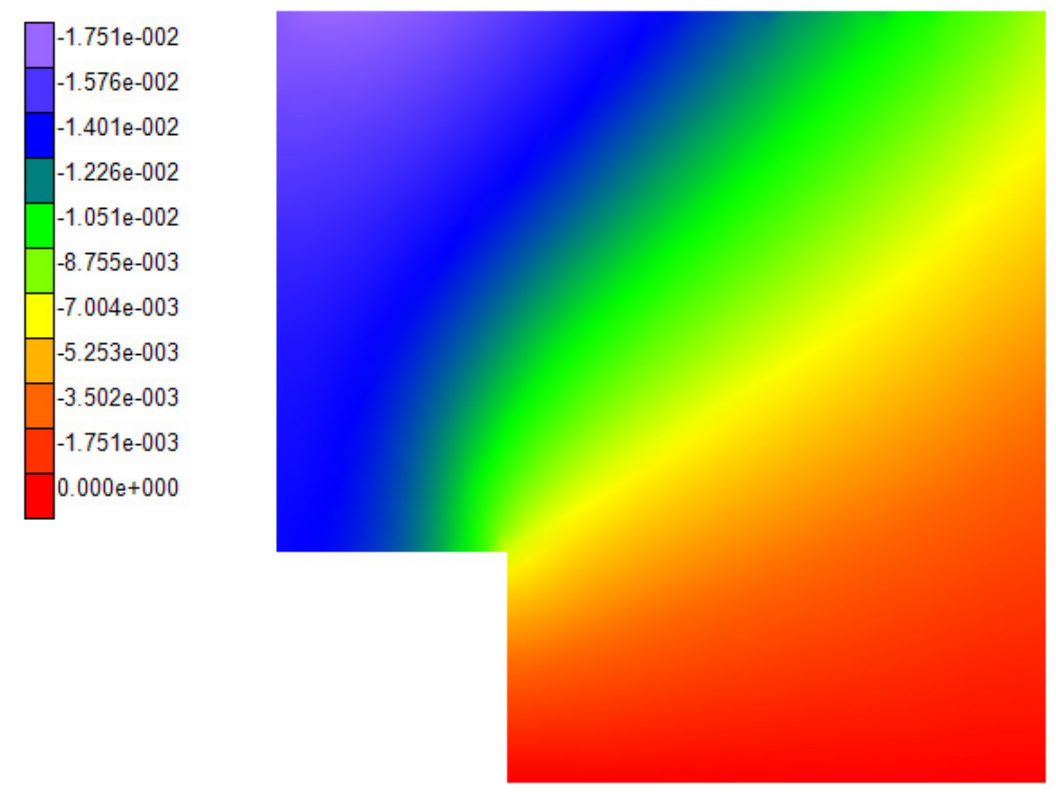

(b) DLSM

Figure 7. Contour plot of the y-direction displacement results for the square hole problem with $E=10^{3} \mathrm{MPa}$ and $v=0.2$ 


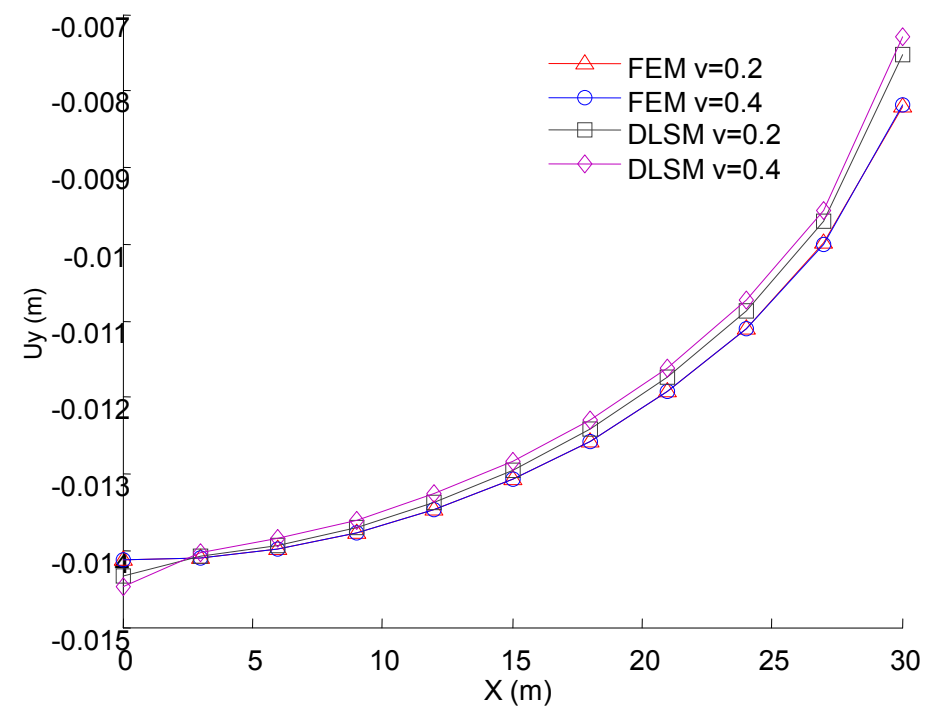

Figure 8. The y-direction displacement along the top surface of the square hole with $E=10^{3} \mathrm{MPa}$ and two different Poisson's ratios. 


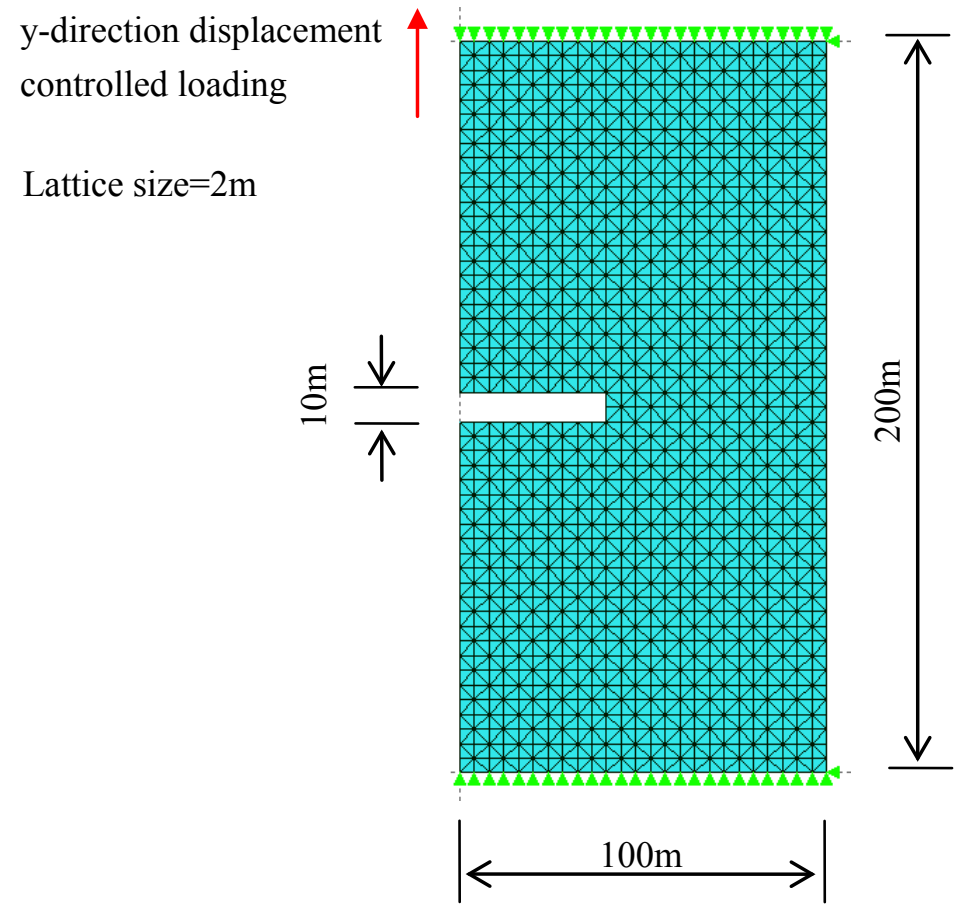

Figure 9. The geometry and boundary conditions for the fracture simulation of a notched specimen under uniaxial tensile loading. 


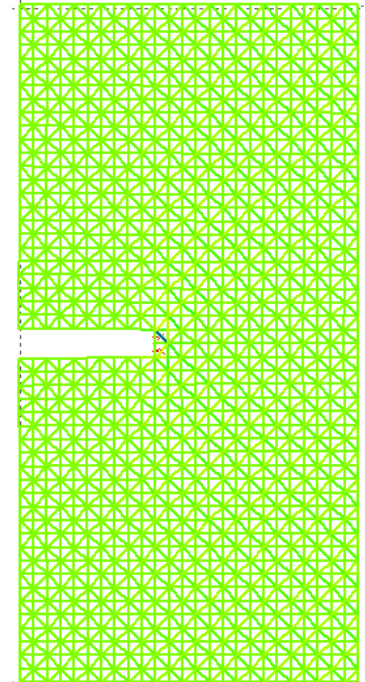

(a) step $=50$

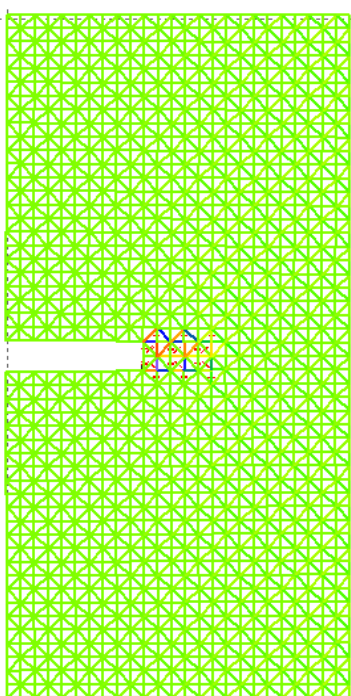

(b) step $=60$

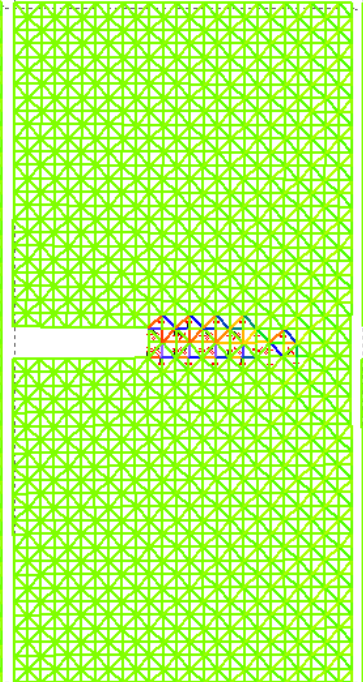

(c) step $=70$

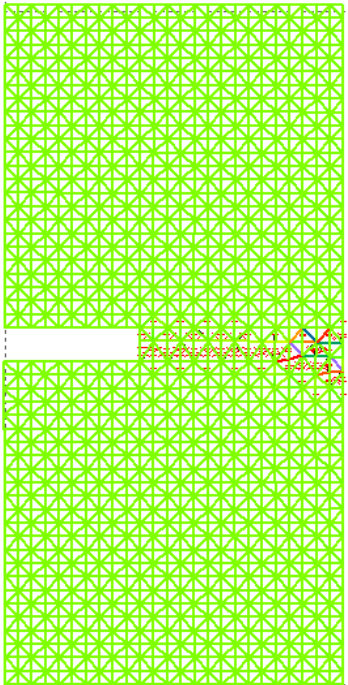

(d) $\mathrm{step}=100$

Figure 10. The fracture process predicted by DLSM. 

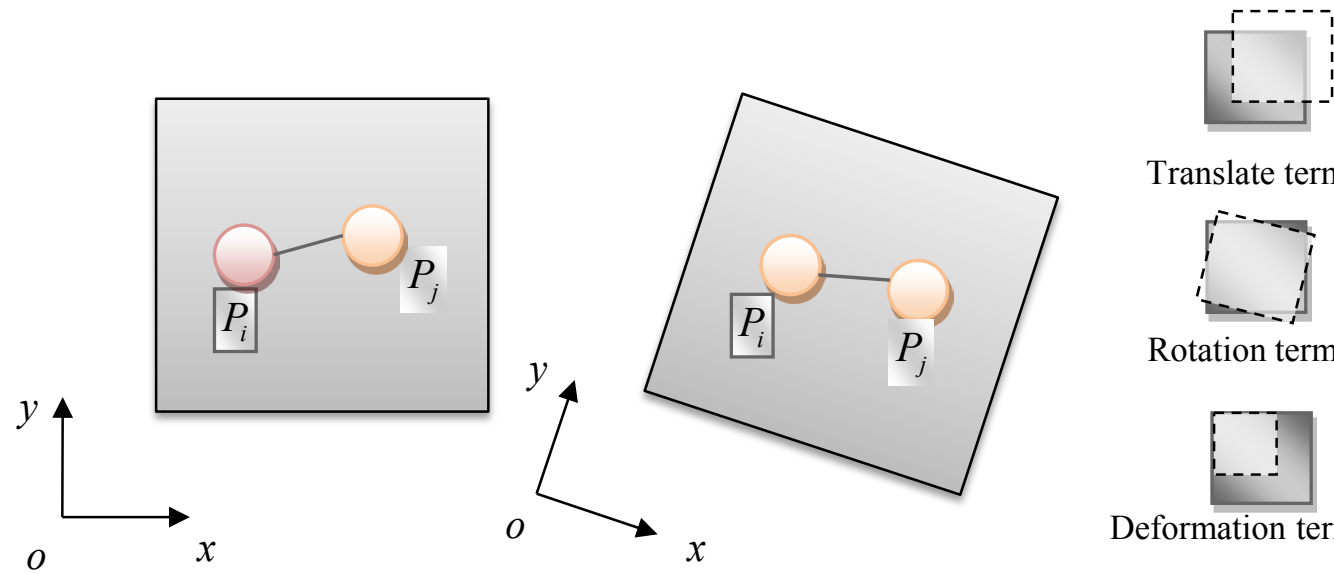

Translate term

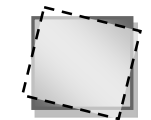

Rotation term

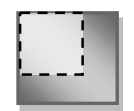

Deformation term

Figure A1. Illustration of the deformation of a cubic unit with a bond connecting two particles. 


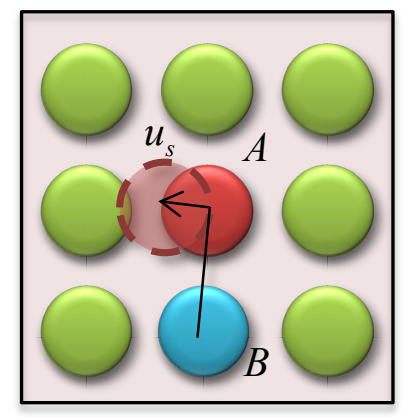

(a) Molecular model of material

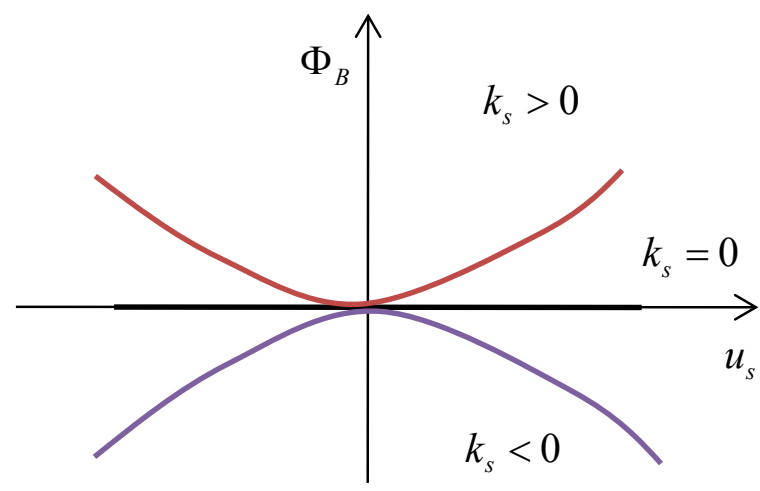

(b) Potential variation at molecular B

Figure B1. Physical explanation of the negative stiffness of shear spring. 


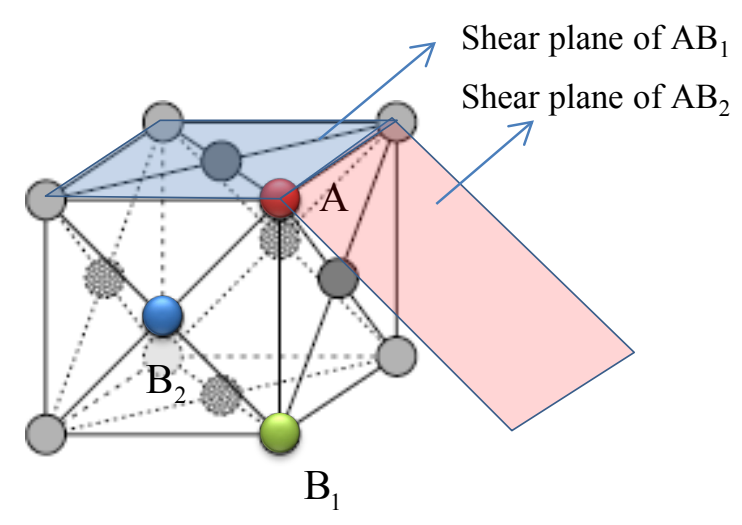

Figure B2. The cubic face-centered lattice (fcc) structure of silver. 


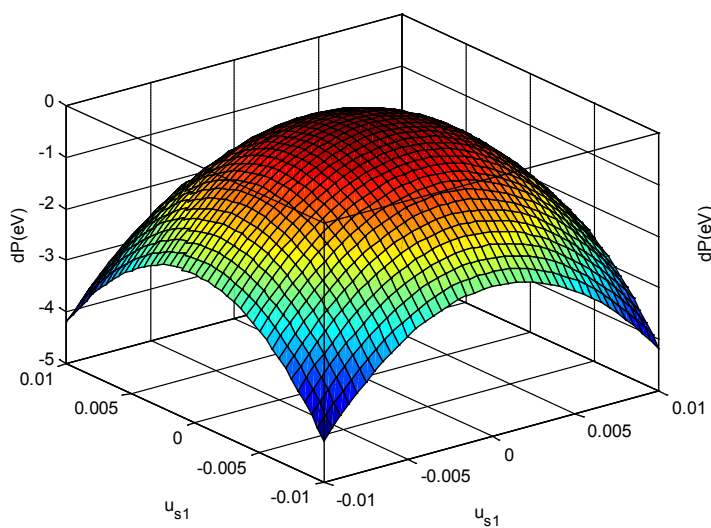

(a) $B_{1}$ atom

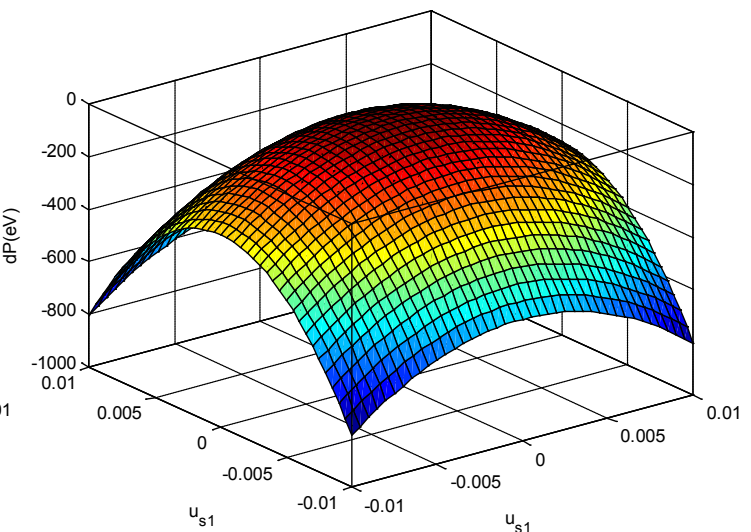

(b) $\mathrm{B}_{2}$ atom

Figure B3. The variation of potential energy at two different atom positions in the case of silver. 
Table 1. Material constants, model parameters and numerical errors of CLSM and DLSM compared with FEM results for the beam bending problem.

\begin{tabular}{ccccccc}
\hline$E(\mathrm{MPa})$ & $v$ & $\alpha^{2 D}$ & $k_{\mathrm{n}}(\mathrm{MN} / \mathrm{m})$ & $k_{\mathrm{s}}(\mathrm{MN} / \mathrm{m})$ & Err_1 (\%) & Err_2 (\%) \\
\hline 10000 & 0.1000 & 3.6447 & 6097.1021 & 3879.9741 & 88.2 & 2.8 \\
10000 & 0.2000 & 3.6447 & 6859.2398 & 2286.4133 & 84.8 & 1.5 \\
10000 & 0.3000 & 3.6447 & 7839.1313 & 603.0101 & 71.2 & 2.1 \\
10000 & 0.3333 & 3.6447 & 8231.0878 & 0.0 & 2.5 & 2.0 \\
\hline
\end{tabular}


Table 2. Material constants, model parameters and numerical errors of DLSM for the beam bending problem using different lattice structures.

\begin{tabular}{ccccccc}
\hline Structure & $E(\mathrm{MPa})$ & $v$ & $\alpha^{2 D}$ & $k_{\mathrm{n}}(\mathrm{MN} / \mathrm{m})$ & $k_{\mathrm{s}}(\mathrm{MN} / \mathrm{m})$ & Err_2 (\%) \\
\hline $\mathrm{a}$ & 10000 & 0.2000 & 3.5596 & 7023.1976 & 2341.0659 & 6.01 \\
$\mathrm{~b}$ & 10000 & 0.2000 & 4.0750 & 6134.9693 & 2044.9898 & 6.23 \\
$\mathrm{c}$ & 10000 & 0.2000 & 3.6447 & 6859.2398 & 2286.4133 & 1.46 \\
$\mathrm{~d}$ & 10000 & 0.2000 & 4.2213 & 5922.3862 & 1974.1287 & 3.11 \\
\hline
\end{tabular}


Table B1. The set of parameters of Finnis-Sinclair potential for silver.

\begin{tabular}{cccccc}
\hline$m$ & $n$ & $\varepsilon$ & $\sigma$ & $c$ & lattice spacing \\
\hline 6 & 12 & $2.5415 \times 10^{-3} \mathrm{eV}$ & $4.09 \AA ̊$ & 144.41 & $1.21875 \AA$ \\
\hline
\end{tabular}

